\title{
HETEROSIS AND COMBINING ABILITY IN A DIALLEL CROSS OF OKRA (Abelmoschus esculentus (L.) MOENCH)
}

\author{
C. DABANDATA, M. J. BELL, A. AMOUGOU and B. H. NGALLE
}

Genetic and Plant Breeding Unit, Department of Plant Biology, Faculty of Science ; University of Yaoundé I, P.O. Box 812 Yaoundé, Cameroon. E-mail : josmarbell@yahoo.fr

\begin{abstract}
A diallel analysis on 8 pure lines of Abelmoschus esculentus was done at 3 sites in Cameroon (Dibang, Yaoundé and Yagoua) in a randomized complete block design, with 3 replications, to estimate both the heterosis and the combining ability. The heterosis over mid-parent and the combining abilities were significant for all the studied traits. Reciprocal effects were observed for all traits, except for the $50 \%$ flowering day. The best results of heterosis, over mid-parent, were recorded by combination $1 \times 2$ for seeds number per pod parameter $(54.78 \%)$. These parameters are supposed to improve the characters in okra.
\end{abstract}

Key-words : Okra, heterosis, diallel analysis.

\section{RESUME}

HETEROSIS ET APTITUDE A LA COMBINAISON EN CROISEMENT DIALLELE CHEZ LE GOMBO (Abelmoschus esculentus (L.) $M O E N C H)$

Une analyse diallèle a été menée dans 3 localités du Cameroun (Dibang, Yaoundé et Yagoua) sur 8 lignées de Abelmoschus esculentus, selon un dispositif en blocs complets randomisés, à 3 répétitions. Les résultats montrent que tous les caractères étudiés ont présenté une hétérosis moyenne et une aptitude aux combinaisons significatives. Des effets réciproques existent chez ces caractères, excepté la date à $50 \%$ de floraison. Les meilleurs résultats de l'hétérosis ont été enregistrés, avec la combinaison $1 \times 2$, pour le nombre de graines par gousse (54,78\%). Ces deux paramètres favoriseraient l'amélioration des caractères chez le gombo.

Mots-clés : Gombo, hétérosis, analyse diallèle.

\section{INTRODUCTION}

Abelmoschus esculentus, is an autogamous crop of the Malvaceae family. The great importance of this crop in the nutrition of populations in many countries throughout the world militate in favor of its improvement. However, this requires the ability to select higherperforming individuals from a population (Murtaza, 2005). That leads to a study of both combining ability and heterosis characteristics. A knowledge of the combining ability can help to identify the best parents that can be hybridized amongst them to use the heterosis or to accumulate some genes fixable through a selection process (Metha et al., 2007).
With regard to the principle, the autogamous crops lead to the selection of pure lines and the phenomenon of heterosis is only important for the allogamous species (Schnell and Becker, 1986). But, it happens that, for some autogamous species, certain level of heterosis, occurs justifying the existence of hybrid varieties. Hybrids show evidence of qualities that cannot be obtained by selection within a species (Wright, 1964). Hybrid vigor (heterosis) occurs relatively frequently in the F1's generation. In particular, when species are exotic or when one experiments on habitats altered by man.

Several studies have been conducted on heterosis while achieving hybridizations between distant crops of okra. The results, although often 
disappointing are very variable. However, some crossing between varieties originated from the United States and Malaysia showed good hybrid vigour (Hamon and Charrier, 1997).

It is known that, in the active region spaning from Guinea to Cameroon, there is a multiplicity of varieties of okra (Hamon and Charrier, 1997). The existence of a considerable range of varieties in this region should be an asset to optimize the research of heterosis in Abelmoschus esculentus and to identify superior individuals with good combining abilities.

\section{MATERIAL AND METHODS}

Experiments were initiated in the year 2004 at the University of Yaoundé $I$ and at Dibang and Yagoua localities. Seeds were obtained from the market, for exotic varieties and from field and local markets for local varieties. Plants were grown three times and self-pollinated to obtain pure lines. Three seeds from pure line parents were then sown in whole at $70 \mathrm{~cm}$ between and within rows and thinned to two plants per hole after plants reached 3 leaf stage. The experimental design was a randomised complete block design, with three replications. Each plot consisted in three rows and each row contain six plants.

The plants were supplemented with a daily artificial watering in the dry season. Crosses were attempted among the 8 pure lines parents to obtain 56 F1 (direct and reciprocal) crosses.

\section{DATACOLLECTION}

The data was collected separately at the 3 sites. Three plants per plot, 8-9 plants were chosen at random and the following characters assessed : number of fruits per plant, plant height, stem basal diameter.

Number of seeds, length of fruit peduncle, fruit diameter and length was counted randomly on a number of 9 fruits retained on the plants of the 3 repetitions on account of 3 per plot. The basal stem diameter was determined for every treatment.

In order to take the weight 3 shares of 100 seeds were randomly selected from the nine retained fruits on the studied genotypes and weighted.
Fruit peduncle and fruit lengths were measured. The $50 \%$ flowering day was considered as the day when half of 18 plants ( 9 of them) flowered in the 3 plots. Estimates of general and specific combining ability variances were obtained as follows, using the diallel analysis described by Griffing as model I, where parents, F1's and reciprocals were included (Griffing, 1956).

For the general combining ability :

$\mathrm{GCA}_{\mathrm{i}}=\left(\tilde{\mathrm{Y}}_{{ }_{\mathrm{o}}}+\tilde{\mathrm{Y}}_{\mathrm{o}}\right) / 2-\tilde{\mathrm{Y}}_{\mathrm{oo}}$, with,

$\mathrm{GCA}_{\mathrm{i}}=$ general combining ability of the $\mathrm{i}$ line ;

$\tilde{Y}_{i o}=$ sum between the averages of the $i$ line, as male parent ;

$\tilde{Y}_{i}=$ sum between the averages of the $i$ line, as female parent;

$\tilde{Y}_{o o}=$ general average of the test.

The SCA were calculated for the hybrids reciprocal F1 from the following equation :

$\operatorname{SCA}_{i j}=\left(\tilde{Y}_{i j}+\tilde{Y}_{j i}\right) / 2-\tilde{Y}_{o o}-G C A_{i}-G C A_{j}$, where

SCAij $=$ specific combining ability of the $i$ and $j$ lines ;

$\tilde{Y}_{\mathrm{ij}}=$ average of the $\mathrm{i}$ line, as male parent, and $\mathrm{j}$, as female ;

$\tilde{\mathrm{Y}}_{\mathrm{ji}}=$ average of the $\mathrm{i}$ line as female parent and $\mathrm{j}$ as male ;

$\tilde{\mathrm{Y}}_{\mathrm{oo}}=$ general average of the test ;

$\mathrm{GCA}_{i}=$ general combining ability of the $i$ line ;

$\mathrm{GCA}_{\mathrm{j}}=$ general combining ability of the $\mathrm{j}$ line .

Reciprocal effects were given by the following equation:

$E M_{i j}=Y_{i j}-Y_{j i} / 2$, where :

$Y_{i j}=$ value of the parent $i$ took as male and $j$ as female;

and $Y_{j i}=$ value of the parent $j$ took as male and $i$ as female.

\section{RESULTS}

The variances due to GCA, SCA and reciprocal effects are presented in Table 1. Variance analysis shows that GCA effects were highly significant for all the traits studied $(P<0.001)$, except for pod peduncle length and $50 \%$ flowering day $(P<0.01$ and $P<0.05$, 
respectively). On the other hand, the SCA effects are highly significant for all the traits.

The estimates of the GCA effects for the 8 parents are presented in Table 2. These results show that Indiana and Clemson Spineless are two parents, whith the highest positive GCA for plant height parameter, with values of 19.15 and 7.05 , respectively. The local varieties 3 and 4 , with values of -10.00 and -13.50 , respectively presented for this same parameter that was significant, but with negative values. No variety presented a positive and significant GCA for all the studied characters.

Table 1 : Analysis of variance on the combining ability for the traits studied.

Analyse de variance sur l'aptitude à la combinaison des caractères étudiés.

\begin{tabular}{|c|c|c|c|c|}
\hline \multirow{2}{*}{ Traits } & \multicolumn{4}{|c|}{ Mean square for each parameter } \\
\hline & GCA & SCA & $\mathrm{RE}$ & Error \\
\hline $\mathrm{PH}$ & $1770.95 * * *$ & $11255.580 * * *$ & $288.420964 * * *$ & 45.32428571 \\
\hline HSW & $0.498601786^{* * *}$ & $69.8671474 * * *$ & $0.531 * * *$ & 0.06517 \\
\hline PPL & $0.325342857^{* *}$ & $14.92908371^{* * *}$ & $0.057717857 * * *$ & 0.010191485 \\
\hline PL & $661.6649875^{* * *}$ & $638.683739^{* * *}$ & $5.43858928 * * *$ & 0.7158127 \\
\hline SNP & $1258.587825 * * *$ & $11337.99681 * * *$ & $119.84885^{* * *}$ & 2.8671428 \\
\hline PG & $1.72824821^{* * *}$ & $15.5673558 * * *$ & $0.1080035^{* * *}$ & 0.016978 \\
\hline PNP & $0.45640982^{* * *}$ & $12.53551615^{* * *}$ & $0.234717857 * *$ & 0.07222857 \\
\hline SD & $0.3259375^{* * *}$ & $3.13530133^{* * *}$ & $0.10264285^{* * *}$ & 0.007142857 \\
\hline FD & $40.6316571^{*}$ & $5779.9853^{* * *}$ & 6.008657 & 12.54129 \\
\hline
\end{tabular}

GCA : general combining ability/aptitude générale à la combinaison ; SCA : specific combining ability/aptitude spécifique à la combinaison : RE : reciprocal effect/effet réciproque ; SD : stem diameter/diamètre au collet : FNP : pod number per plant/nombre de gousses par plante; PG : pod girth/grosseur de la gousse ; SNP : seeds number per pod/nombre de graines par gousse ; PL : pod length/longueur de la gousse; PPL : pod peduncle length/longueur du pédoncule de la gousse; HSW : 100 seeds weight/masse de 100 graines ; FD : 50 \% flowering day/jour de $50 \%$ de floraison ; $\mathrm{PH}$ : plant height/taille de la plante 
Table 2 : General combining ability estimates of the 8 parents.

Valeurs de l'Aptitude Générale à la Combinaison des huit parents.

\begin{tabular}{|c|c|c|c|c|c|c|c|c|c|}
\hline \multirow{2}{*}{ Traits } & \multicolumn{9}{|c|}{ CGA per genotype } \\
\hline & $\mathrm{C}$ & I & $\mathrm{T}$ & 1 & 2 & 3 & 4 & 5 & LSD \\
\hline $\mathrm{PH}$ & 7.050 & 19.15 & 0.14 & 0.825 & 4.04 & -10.005 & -13.50 & -7.67 & 3.72 \\
\hline HSW & -0.095 & 0.19 & 0.26 & -0.135 & -0.03 & 0.05 & -0.285 & 0.045 & 0.125 \\
\hline PPL & -0.11 & 0.035 & -0.02 & -0.115 & 0.19 & 0.06 & -0.20 & 0.19 & 0.049 \\
\hline PL & -3.57 & -0.29 & 1.94 & -1.00 & 4.53 & -1.765 & 0.265 & -0.085 & 0.41 \\
\hline SNP & -0.23 & 11.12 & 2.46 & 2.9 & 11.09 & -3.93 & -11.85 & -11.56 & 0.83 \\
\hline PG & 0.55 & 0.215 & -0.04 & 0.215 & -0.01 & -0.17 & -0.285 & -0.495 & 0.06 \\
\hline PNP & -0.195 & 0.135 & -0.055 & -0.195 & -0.105 & 0.115 & 0.265 & 0.055 & 0.132 \\
\hline SD & 0.045 & 0.215 & 0.155 & 0.008 & 0.005 & -0.125 & -0.175 & -0.145 & 0.041 \\
\hline FD & -0.18 & -2.035 & 0.33 & 1.19 & 2.64 & -2.21 & 0.42 & -0.145 & 1.74 \\
\hline
\end{tabular}
gousse : SNP : seeds number per pod/nombre de graines par gousse; PL : pod length/longueur de la gousse; PPL : pod peduncle length/ longueur du pédoncule de la gousse ; HSW : 100 seeds weight/masse de 100 graines ; FD : $50 \%$ flowering day/jour de $50 \%$ de floraison; PH : plant height/taille de la plante ; C : clemson spineless ; I : Indiana ; T : Rouge de Thiès ; L1 : local variety $1 /$ variété locale

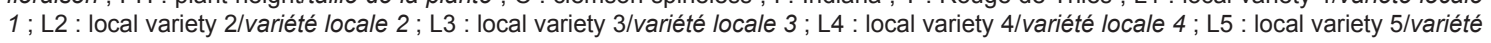
locale 5.

Specific combining ability (SCA) effects of the F1 hybrids for all characters showed that the hybrid $4 \times 5$ possesses a positive SCA, for all traits, on the contrary, the Cxl combination only had negative values (Table 3). Positive significant effects of the SCA have been observed for nearly all parameters in some hybrid combinations.

Heterosis values for the hybrids evaluated at the 3 sites varied largely from negative to positive. The highest value was recorded for $5 \times 4$ combination for seeds number per pod parameter with the value of $54.78 \%$. This combination was followed, respectively, for plant height and stem diameter by the $1 \times 3(40.89 \%)$ and $1 \times 4$ (34.94\%) hybrids. Eight combinations ( $1 \times 3,2$ $\times 5,2 \times \mathrm{T}, 3 \times 5,5 \times 3,5 \times 4,5 \times I$ and $\mathrm{T} \times \mathrm{I}$ ) exhibited heterosis for both important traits needed by breeders, such as : pod girth and pod length (Table 4). All the 3 best combinations were provided from local parents.

Estimates of the reciprocal effects show that they exist for all traits except the $50 \%$ flowering day (Table 5). Among hybrids, the combination $3 \times 4$ exhibited the higher significant positive reciprocal effects (18.51) for the seed number per pod parameter. 
Table 3 : Specific combining ability (SCA) estimates of the F1 hybrids.

Aptitude Spécifique à la Combinaison des hybrides F1.

\begin{tabular}{|c|c|c|c|c|c|c|c|c|c|}
\hline \multirow{2}{*}{ Crosses } & \multicolumn{9}{|c|}{ Genotypes } \\
\hline & SD & FNP & PG & SNP & PL & PPL & HSW & FD & $\mathrm{PH}$ \\
\hline L1x L 2 & -0.09 & -0.365 & -0.33 & 18.195 & 1.365 & 0.08 & 0.57 & -0.27 & -0.4950 \\
\hline L1x L 3 & 0.135 & 0.325 & 0.205 & 16.34 & 1.735 & 0.34 & -0.555 & -0.86 & 20.42 \\
\hline L1x L 4 & 0.097 & -0.205 & 0.26 & -15.345 & -2.715 & -0.22 & -0.16 & -1.215 & -3.635 \\
\hline L1x L5 & -0.105 & -0.015 & -0.155 & -9.295 & -1.06 & -0.295 & 0.285 & 2.52 & -15.465 \\
\hline $\mathrm{L} 1 \mathrm{xC}$ & -0.06 & -0.605 & -0.13 & -0.530 & 0.645 & -0.155 & 0.065 & 0 & -1.675 \\
\hline L1xI & 0.1 & 0.075 & -0.145 & 1.740 & 0.66 & -0.01 & 0.11 & -1.98 & 1.76 \\
\hline $\mathrm{L} 1 \mathrm{xT}$ & -0.02 & 0.45 & -0.385 & -6.270 & -0.83 & -0.08 & 0.085 & 0.82 & -5.51 \\
\hline L2x L3 & 0.09 & -0.175 & -0.15 & -5.955 & -0.935 & -0.005 & 0.385 & -0.2 & -13.435 \\
\hline L2x L4 & -0.083 & -0.245 & -0.040 & 4.895 & 0.180 & 0.105 & -0.425 & 0 & -8.05 \\
\hline L2x L5 & 0.19 & 0.285 & 0.22 & 7.6 & 0.06 & 0.16 & -0.21 & -0.655 & 13.7 \\
\hline $\mathrm{L} 2 \mathrm{xC}$ & -0.10 & -0.01 & -0.125 & -1.75 & 1.235 & -0.015 & 0.065 & $-1,615$ & -8 \\
\hline L2xI & 0.04 & 0,175 & -0.025 & -1.78 & 1.305 & -0.005 & 0.245 & 1.01 & 1.18 \\
\hline L2xT & 0.035 & -0.155 & 0.25 & -0.175 & -0.575 & 0.17 & -0.32 & 0.645 & 0.165 \\
\hline L3x L4 & -0.153 & -0.05 & -0.020 & 0.485 & -0.92 & -0.1 & 0.25 & -0.925 & 2.255 \\
\hline L3x L5 & -0.365 & 0.25 & -0.045 & -2.5 & 0.7 & -0.185 & 0.15 & 1.75 & -12.98 \\
\hline $\mathrm{L} 3 \mathrm{xC}$ & -0.065 & 0.125 & 0.025 & -2.96 & 0.455 & -0.015 & -0.38 & 0.51 & 2.030 \\
\hline L3xI & -0.165 & -0.08 & 0.06 & -1.505 & -1.135 & -0.165 & -0.39 & 1.085 & 0.395 \\
\hline L3xT & -0.105 & -0.02 & 0.03 & -3.89 & 0.15 & 0 & -0.28 & -2.11 & 5.935 \\
\hline L4x L5 & 0.122 & 0.5 & 0.03 & 17.19 & 1.95 & 0.145 & 0.675 & -0.1 & 7.060 \\
\hline $\mathrm{L} 4 \mathrm{xC}$ & 0.042 & 0.155 & -0.195 & 4.225 & 0.695 & 0.055 & 0.135 & 0.21 & 3.07 \\
\hline L4xI & 0.127 & -0.275 & 0.095 & 1.02 & 1.4 & 0.195 & 0.115 & 0.845 & -2.03 \\
\hline $\mathrm{L} 4 \mathrm{xT}$ & -0.073 & -0.155 & -0.305 & -9.70 & 0.335 & -0.105 & -0.005 & -0.02 & -8.435 \\
\hline L5 $\mathrm{xC}$ & 0.095 & -0.505 & 0.04 & 3.04 & -0.055 & -0.035 & -0.57 & -0.445 & 5.41 \\
\hline L5xI & -0.04 & -0.33 & 0.06 & 4.27 & -1.25 & -0.01 & 0.26 & -0.98 & 6.845 \\
\hline L5xT & 0.14 & -0.32 & 0.025 & 3.65 & 1.13 & -0.04 & -0.205 & -2.18 & 0.52 \\
\hline CxI & -0.035 & -0.64 & -0.075 & -2.17 & -0.855 & -0.095 & -0.075 & 1.5 & -6.665 \\
\hline $\mathrm{CxT}$ & 0.03 & -0.435 & 0.16 & 5.685 & -1.235 & -0.18 & 0.415 & 0.8 & 1.38 \\
\hline IxT & 0.025 & 0.03 & 0.04 & 3.70 & 0.385 & -0.1 & 0.275 & -0.12 & 4.78 \\
\hline LSD & 0.11 & 0.35 & 0.17 & 2.22 & 1.11 & 0.13 & 0.33 & 4.65 & 8.61 \\
\hline
\end{tabular}

SD : stem diameter/diamètre au collet ; FNP : pod number per plant/nombre de gousses par plante ; PG : pod girth/grosseur de la gousse ; SNP : seeds number per pod/nombre de graines par gousse ; PL : pod length/longueur de la gousse ; PPL : pod peduncle length/ longueur du pédoncule de la gousse; HSW : 100 seeds weight/masse de 100 graines ; FD : $50 \%$ flowering day/jour de $50 \%$ de floraison ; PH : plant height/taille de la plante ; C : clemson spineless ; I : Indiana, T : rouge de Thiès, L1 : local variety 1/variété locale 1 ; L2 : local variety $2 /$ variété locale 2 ; L3 : local variety $3 /$ variété locale 3 ; L4 : local variety 4/variété locale 4 ; L5 : local variety $5 /$ variété locale $5 ;$ LSD : least significant difference/plus petite différence significative. 
Table 4 : Heterosis (\%) estimates of the F1 hybrids.

Evaluation de l'hétérosis (\%) des hybrids F1.

\begin{tabular}{|c|c|c|c|c|c|c|c|c|c|}
\hline \multirow{2}{*}{ Crosses } & \multicolumn{9}{|c|}{ Traits } \\
\hline & SD & PNP & PG & SNP & $\mathrm{PL}$ & PPL & HSW & $\mathrm{PH}$ & FD \\
\hline L1x L2 & 8.22 & -33.68 & -11.08 & 35.32 & 17.07 & 7.27 & 7.95 & -6.93 & 1.04 \\
\hline L1x L3 & 4.32 & 20.27 & 5.56 & 30.46 & 15.37 & 9.96 & -15.11 & 40.89 & -4.66 \\
\hline L1x L4 & 34.94 & -12.99 & -2.75 & -20.88 & -22.15 & -11.49 & 0.39 & 5.69 & -3.95 \\
\hline L1x L5 & -18.90 & -10.25 & -14.84 & 0.25 & -8.79 & -25.77 & 2.05 & -45.2 & 4.61 \\
\hline $\mathrm{L} 1 \mathrm{xC}$ & -0.42 & -42.29 & -13.78 & 3.8 & 19.06 & -12.83 & 5.34 & -0.39 & -1.42 \\
\hline L1xI & 14.81 & -23.81 & -13.66 & 1.07 & 1.29 & -3.77 & 5.19 & -3.78 & -1.99 \\
\hline L1xT & 8.37 & 8.48 & -23.15 & -11.83 & -10.26 & -15.15 & 10.46 & -10 & 0.48 \\
\hline L2x1 & -15.07 & -19.30 & -28.77 & 53.17 & 25.09 & 7.66 & 25.65 & -11.8 & -5.41 \\
\hline L2x L3 & -35.09 & -25.48 & -6.43 & 13.55 & 7.91 & 7.3 & -4.56 & -25.92 & -3.23 \\
\hline L2x L4 & -16.73 & -24.16 & -6.84 & 17.81 & 16.86 & 8.78 & 5.02 & -24.05 & -1.26 \\
\hline L2x L5 & 4.20 & -24.56 & 16.45 & 38.59 & 8.59 & 5.34 & -0.79 & -3 & -2.66 \\
\hline $\mathrm{L} 2 \mathrm{xC}$ & -7.75 & -30.54 & -10.32 & 22.47 & 23.84 & -1.63 & 15.76 & -20.26 & -3.06 \\
\hline L2xI & 20.97 & -9.96 & -5.63 & 27.32 & 24.42 & 0.63 & 21.09 & -7.61 & 2.05 \\
\hline L2xT & 2.70 & -14.20 & 6.34 & 20.57 & 13.15 & 18.53 & -3.94 & 2.75 & -4.97 \\
\hline L3x L1 & -22.77 & 13.51 & -11.39 & 21.49 & 5.96 & -0.71 & -9.67 & 9.8 & -1.75 \\
\hline L3x L2 & -25.07 & 9.89 & -8.73 & -1.47 & -2.99 & 8.88 & 8.36 & -12 & -0.8 \\
\hline L3x L4 & -11.31 & 5.67 & 9.6 & 26.74 & -1.9 & 1.15 & 3.02 & 6.56 & -2.42 \\
\hline L3x L5 & -39.59 & 21.62 & 1.71 & 11.63 & 5.12 & -12.52 & -10.01 & -11.8 & 2.66 \\
\hline $\mathrm{L} 3 \mathrm{xC}$ & -25.77 & -16.14 & -1.3 & 13.65 & 21.7 & -9.57 & -4.79 & 13.66 & -3.35 \\
\hline L3xI & -21.45 & -13.34 & 10.52 & 2.79 & -11.87 & -8.71 & -6.59 & 9.27 & 4.29 \\
\hline L3xT & -27.39 & -3.45 & -0.95 & -7.74 & -1.43 & -10.4 & -8.08 & 15.77 & -10.63 \\
\hline L4x1 & -11.65 & -28.25 & -7 & -11.52 & -15.34 & -13.41 & 12.43 & -30.06 & -4.15 \\
\hline L4x L2 & 13.17 & -19.58 & -8.59 & 27.36 & 10.59 & 27.19 & -4.11 & -14.45 & -2.61 \\
\hline L4x L3 & -39.88 & -4.31 & -15.23 & -21.79 & -2.69 & -9.23 & 0.57 & -7.28 & -4.74 \\
\hline L4x L5 & -10.29 & -3.92 & -11.4 & 28.99 & 5.14 & 0.87 & 22.9 & -7.55 & 2.43 \\
\hline $\mathrm{L} 4 \mathrm{xC}$ & 3.51 & -28.41 & -11.51 & 17.37 & 13.25 & -4.59 & 14.26 & 3.11 & 0.2 \\
\hline L4xI & 23.90 & -24.69 & 3.72 & 7.09 & 25.88 & 11.48 & 14.11 & 5.29 & 0.96 \\
\hline $\mathrm{L} 4 \mathrm{xT}$ & -10.19 & -27.53 & -15.56 & -19.34 & 8.46 & -8.72 & 12.68 & -26.81 & -0.69 \\
\hline L5x L1 & 5.51 & -9.02 & -9.57 & 14.5 & 4.06 & -11.18 & 22.95 & 9.16 & 2.6 \\
\hline L5x L2 & 26.57 & 23.51 & -5.59 & 44.86 & 12.97 & 16.37 & 5.54 & 20.32 & -1.67 \\
\hline L5x L3 & -39.00 & 13.51 & 2.86 & 13.98 & 7.63 & -10.89 & 7.01 & -10.43 & 2.45 \\
\hline L5x L4 & 36.63 & 28.25 & 10.74 & 54.78 & 26.69 & 4.35 & 20.61 & 19.35 & -5.09 \\
\hline $\mathrm{L} 5 \mathrm{xC}$ & 6.44 & -40.88 & -0.37 & 28.6 & 4.55 & -13.77 & -4.75 & -4.55 & 1.68 \\
\hline L5xI & 4.76 & -28.42 & 2.47 & 27.13 & 1.46 & -12.52 & 10.39 & 39.68 & -3.4 \\
\hline L5xT & 8.60 & -20.14 & -0.73 & 19.59 & 10.28 & -7.35 & -1.4 & 19.18 & -5.16 \\
\hline Cx L1 & -15.48 & -53.89 & -20.87 & 10.91 & -1.75 & -21.05 & -2.24 & -16.08 & 1.42 \\
\hline Cx L2 & -10.70 & -29.53 & -15.48 & 12.3 & 20.6 & 4.88 & -14.12 & -18.02 & -2.86 \\
\hline Cx L3 & -26.38 & -20.66 & -5.4 & -14.24 & -10.47 & -8.58 & -24.24 & -6.91 & 5.73 \\
\hline Cx L4 & 0.88 & -24.15 & -17.99 & 7.62 & 10.33 & -2.47 & -5.81 & -14.31 & 0.2 \\
\hline Cx L5 & 2.15 & -44.55 & -3.35 & 26.25 & 7.39 & -8.02 & -14.6 & 21.69 & -1.68 \\
\hline CxI & -12.82 & -42.07 & -6.02 & 5.25 & -11.14 & -9.76 & 1.62 & -13.94 & 7.6 \\
\hline CxT & 3.88 & -37.83 & -6.18 & 0.8 & -23.2 & -13.26 & 3.57 & 6.45 & 1.72 \\
\hline Ix L1 & 9.26 & -12.44 & -16.01 & 23.49 & 10.53 & -14.34 & 18.36 & 11.72 & -4.74 \\
\hline Ix L2 & -8.06 & -21.15 & -5.28 & 11.55 & 12.04 & 14.95 & 4.59 & 0.15 & 2.25 \\
\hline Ix L3 & -39.27 & -12.03 & -3.09 & 0.95 & 3.23 & -13.64 & -11.43 & 12.26 & 1.36 \\
\hline Ix L4 & 10.24 & -32.41 & -5.13 & 9.96 & 0.66 & 2.46 & 13.5 & -17.2 & 2.65 \\
\hline Ix L5 & -8.57 & -27.19 & 7 & 39.1 & -3.95 & -1.54 & 17.65 & 3.42 & 2.06 \\
\hline $\mathrm{IxC}$ & -1.54 & -54.02 & -13.25 & 7.36 & 9.3 & -16.38 & -0.9 & -9.34 & 2.98 \\
\hline IxT & 0.55 & -36.08 & -10.25 & -1.56 & 1.05 & -12.31 & 7.25 & 1.83 & -6.79 \\
\hline Tx L1 & -11.01 & -8.48 & -28.01 & -12.12 & -7.09 & -10.69 & -1.06 & -12.12 & -3.04 \\
\hline Tx L2 & 5.02 & -28.09 & -4.66 & 0.43 & -7.67 & 0 & -2.1 & -19.66 & 1.7 \\
\hline Tx L3 & -26.75 & -4.98 & -2.25 & -14.45 & 0.26 & -4 & -13.96 & 8.16 & -2.72 \\
\hline Tx L4 & 0.93 & -14.39 & -22.57 & -16.57 & -2.41 & -6.67 & -3.44 & -11.66 & -5.25 \\
\hline Tx L5 & 15.84 & -27.92 & -0.73 & 18.55 & 4.17 & -12.06 & -0.35 & -11.8 & -6.77 \\
\hline TxC & -9.71 & -47.26 & -2.4 & 16.3 & 9.14 & -19.82 & 3.24 & -11.24 & -0.92 \\
\hline TxI & 6.01 & -6.45 & 2.98 & 12.86 & 2.69 & -10.96 & 12.27 & 27.79 & 4.86 \\
\hline
\end{tabular}

SD : stem diameter/diamètre au collet ; FNP : pod number per plant/nombre de gousses par plante ; PG : pod girth/grosseur de la gousse; SNP : seeds number per pod/nombre de graines par gousse ; PL : pod length/longueur de la gousse ; PPL : pod peduncle length/ longueur du pédoncule de la gousse ; HSW : 100 seeds weight/masse de 100 graines ; FD : $50 \%$ flowering day/jour de $50 \%$ de floraison ; $\mathrm{PH}$ : plant height/taille de la plante ; C : clemson spineless ; I : Indiana ; T : rouge de Thiès ; L1 : local variety $1 /$ variété locale 1 ; L2 : local variety $2 /$ variété locale 2 ; L3 : local variety $3 /$ variété locale 3 ; L4 : local variety 4 /variété locale 4 ; L5 : local variety $5 /$ variété locale 5 . 
Table 5 : Estimates of the reciprocal effects.

Evaluation des effets réciproques.

\begin{tabular}{|c|c|c|c|c|c|c|c|c|c|}
\hline \multirow{2}{*}{ Crosses } & \multicolumn{9}{|c|}{ Traits } \\
\hline & SD & PNP & $\mathrm{PG}$ & SNP & PL & PPL & HSW & $\mathrm{PH}$ & FD \\
\hline L1x L2 & 0.17 & -26.49 & 0.355 & -6.275 & -0.495 & -0.005 & -0.495 & 2.665 & 1.89 \\
\hline L1x L3 & 0.235 & 16.89 & 0.305 & 3.240 & 0.745 & 0.15 & -0.17 & 12.54 & -0.78 \\
\hline L1x L4 & 0.29 & -20.62 & 0.085 & -3.335 & -0.425 & 0.025 & -0.31 & 15.83 & 0.06 \\
\hline L1x L5 & -0.155 & -9.63 & -0.095 & -4.905 & -1.140 & -0.225 & -0.56 & -22.925 & 0.56 \\
\hline $\mathrm{L} 1 \mathrm{xC}$ & 0.090 & -48.09 & 0.130 & -2.240 & 1.220 & 0.125 & 0.22 & 5.89 & -0.78 \\
\hline L1xI & 0.030 & -18.13 & 0.040 & -6.190 & -0.645 & 0.14 & -0.33 & -5.13 & 0.73 \\
\hline L1xT & 0.11 & 0.00 & 0.08 & 0.09 & -0.225 & -0.07 & 0.325 & 0.80 & 1.00 \\
\hline L2x L3 & -0.47 & -7.79 & 0.035 & 5.665 & 0.965 & -0.02 & -0.425 & -6.815 & -0.67 \\
\hline L2x L4 & -0.21 & -21.87 & 0.03 & -3.555 & 0.45 & -0.215 & 0.25 & -5.075 & 0.39 \\
\hline L2x L5 & -0.16 & -0.53 & 0.335 & -2.260 & -0.430 & -0.155 & -0.18 & -11.85 & -0.28 \\
\hline $\mathrm{L} 2 \mathrm{xC}$ & 0.02 & -30.04 & 0.08 & 3.37 & 0.22 & -0.09 & 0.915 & -1.035 & -0.06 \\
\hline L2xI & 0.18 & -15.55 & -0.005 & 4.61 & 0.98 & -0.17 & 0.44 & -3.24 & -0.06 \\
\hline L2xT & -0.015 & -21.14 & 0.15 & 6.535 & 1.670 & 0.265 & -0.055 & 10.425 & -1.95 \\
\hline L3x L4 & 0.24 & 0.68 & 0.375 & 18.515 & 0.07 & 0.135 & 0.075 & 5.33 & 0.62 \\
\hline L3x L5 & -0.005 & 17.57 & -0.015 & -0.87 & -0.29 & -0.025 & -0.54 & -0.5 & 0.06 \\
\hline $\mathrm{L} 3 \mathrm{xC}$ & 0.005 & -18.40 & 0.055 & 9.5 & 2.75 & -0.015 & 0.66 & 6.535 & -2.33 \\
\hline L3xI & 0.135 & -12.69 & 0.165 & 0.555 & -1.46 & 0.065 & 0.145 & -0.815 & 0.72 \\
\hline L3xT & -0.005 & -4.21 & 0.015 & 2.24 & -0.165 & -0.10 & 0.195 & 2.445 & -2.11 \\
\hline L4x L5 & -0.285 & 12.16 & -0.335 & -9.41 & -2.13 & -0.05 & 0.06 & -10.855 & 2.06 \\
\hline $\mathrm{L} 4 \mathrm{xC}$ & 0.015 & -26.28 & 0.1 & 3.275 & 0.2 & -0.03 & 0.57 & 6.22 & 0.00 \\
\hline L4xI & 0.07 & -28.55 & 0.125 & -0.85 & 2.015 & 0.11 & 0.015 & 7.035 & -0.44 \\
\hline L4xT & -0.06 & -20.96 & 0.095 & -0.91 & 0.88 & -0.03 & 0.445 & -5.46 & 1.28 \\
\hline L5xC & 0.025 & -42.72 & 0.04 & 0.76 & -0.27 & -0.095 & 0.29 & -8.815 & 0.89 \\
\hline L5xI & 0.07 & -27.80 & -0.055 & -3.41 & 0.575 & -0.16 & -0.185 & 10.575 & -1.39 \\
\hline L5xT & -0.04 & -24.03 & 0 & 0.33 & 0.655 & 0.08 & -0.03 & 10.52 & 0.45 \\
\hline $\mathrm{CxI}$ & -0.055 & -48.05 & 0.09 & -0.54 & -1.555 & 0.095 & 0.07 & -1.13 & 1.17 \\
\hline $\mathrm{CxT}$ & 0.07 & -42.55 & -0.045 & -4.465 & -2.495 & 0.11 & 0.01 & 5.185 & 0.72 \\
\hline IxT & -0.025 & -21.26 & -0.14 & -3.59 & -0.145 & -0.02 & -0.135 & -6.46 & -3.06 \\
\hline LSD & 0.12 & 0.39 & 0.19 & 2.51 & 1.25 & 0.14 & 0.37 & 9.74 & 5.26 \\
\hline
\end{tabular}

SD : stem diameter/diamètre au collet; FNP : pod number per plant/nombre de gousses par plante ; PG : pod girth/grosseur de la gousse ; SNP : seeds number per pod/nombre de graines par gousse; PL : pod length/longueur de la gousse ; PPL : pod peduncle length/longueur du pédoncule de la gousse; HSW : 100 seeds weight/masse de 100 graines ; FD : $50 \%$ flowering day/jour de $50 \%$ de floraison; PH : plant height/taille de la plante ; LSD : Least Significant Difference/plus petite différence significative ; C : Clemson Spineless ; I : Indiana ; T : Rouge de Thiès ; L1 : local variety $1 /$ variété locale 1 ; L2 : local variety $2 /$ variété locale 2 ; L3 : local variety $3 /$ variété locale 3 ; L4 : local variety $4 /$ variété locale $4 ;$ L5 : local variety $5 /$ variété locale 5.

\section{DISCUSSION}

Significant effects in general and specific combining abilities indicate the importance of both additive and non-additive gene action. These results agreed with reports on plant height (Rajani et al., 2001) and on fruit length (Chaudary et al., 1991). Similar results of significance of variances due to general combining ability were also recorded on fruit girth (Rajani et al., 2001 ;
Chaudary et al., 1991 and Shivagamasundari et al., 1992). These positive results corroborate also those showing that, in okra, the variance of the GCA is meaningful on the first flowering days, the $50 \%$ flowering day and the plant height (Metha et al., 2007).

These results becoming positively widespread for all parameters only confirming the idea that the GCA was in most cases, predominant (Hamon and Charrier, 1997). The significant 
values of the GCA recorded by genotype indicate the possibility to have productive hybrids with various others (Bouharmont, 1995). Genotypes presenting a high and positive GCA for plant height trait, such as Clemson Spineless (7.05) and Indiana (19.15) show a negative GCA for fruit length (Table 2). It shows that these traits are correlated negatively, which would entail some difficulties in a process of improvement of the above traits.

The significant effect of SCA indicates changes between the F1 hybrids. Significant results of the SCA have also been reported for seeds number per pod, fruits diameter and length (Hamon and Charrier, 1997) and for the day of 50 \% flowering (Hamon and Van Sloten, 1995). The performance of the hybrids indicated by this parameter suggests the possibility of improving these characters in the okra improvement program. The SCA of two populations showed differences in the frequencies of the genes and their divergence as compared to the diallel parents (Viana and Matta, 2003). Ttwo pure lines presented a significant SCA means than the hybrid originated from the combination of these two lines was extremely effective. Therefore, according to Griffing (1956) and Machado et al. (2002) these crosses having a high SCA and descents of parents that GCA is also high are efficient in the choice of the most promising hybrid population. It confirms the objective in the analysis of the combining ability because, the analysis of the effects of the GCA leads to the identification of the superior parents (Viana and Matta, 2003). Those parents would finally continue to be self-fertilized to identify by the test of the SCA the pairs of lines which produce the best hybrids (Viana, 2000).

All the three best combinations exhibiting significant heterosis were from local parents. The results show that although these varieties are all local, they remain however genetically distant. Therefore, the heterosis is a special genetic mechanism where by the genotypes of distant origins are brought together in a specific model in order to express their capacity and to change the magnitude of a particular character (Indu et al., 2003). Then, some varieties originated from the same locality can be genetically distant as compared to exotics. A positive heterosis was also observed in diallel crossings for plant height
(Fatokun, 1987 ; Poshiva et Shukla, 1986 ; Kumbhani et al., 1993), pod length, stem diameter, pod diameter. A negative heterosis was found for the number of seeds per pod. The values of hybrids were lower than those of the parents (Fatokun, 1987). Similary, for plant height, a heterosis over the best parent of $-10.33 \%$ was found (Nimbalkar, 2004).

Reciprocal differences observed for traits studied revealed that maternal effects were significant in the inheritance of these characters. Reciprocal differences were also observed for plant height, single fruit weight and fruit yield per plant (Rajani et al., 2001). The reciprocal differences observed for these traits were probably due to cytoplasmic inheritance.

\section{CONCLUSION}

Results of the study show that, combining ability and heterosis were important. Genetic variation for the characters appeared to be influenced predominantly by genes with additive or dominance effects. Seeds per pod is the most heterotic character among the traits studied. Some varieties originated from the same locality were genetically distant, as compared to exotics. Estimates of the reciprocal effects showed no significant difference for the $50 \%$ flowering day implying that the cytoplasmic inheritance did not play any significant role in the expression of this trait. The genetic variability for each of the traits and the importance of both GCA and SCA show that it is possible to improve characters in okra crop. Then hybrids $5 \times 4,2 \times 5,2 \times \mathrm{T}$ and $1 \times 3$ exhibiting good heterosis for both pod girth and pod length can be recommended to breeders.

\section{REFERENCES}

Bouharmont J. 1995. Création variétale et amélioration des plantes. In : Tayeb et Persoons (Eds.). Agronomie moderne. Hatier-AUPELF-UREF, Paris pp. 311 - 338.

Chaudhary R. C., J. Kumar, Vidyasagar and S. K. Sharma. 1991. Hyrid vigor in okra Abelmoschus esculentus (L.) Moench. Himachal J. Agric. Res. 17 (1/2) : 26 - 31.

Fatokun C. A. 1987. Wide hybridization in okra. Theor. Appl. Genet. 74 : 483 - 486. 
Griffing B. 1956. A generalized treatment of the use of diallel crosses in quantitative inheritance. Heredity $10: 31$ - 50 .

Hamon S. et A. Charrier. 1997. Les gombos. In : Charrier, Jacquot, Hamon et Nicolas (Eds.). Amélioration des Plantes Tropicales. CIRAD-ORSTOM, Paris. pp. 313 - 333.

Hamon S. and D. H.Van Floten. 1995. Okra. In : Smartt and Simmonds (Eds.). Evolution of Crop Plants. Longman Scientific \& Technical. London, pp. 350 - 357.

Indu R. C., D.Veeraragavathatham and I. Muthuvel. 2003. Performance of parents and hybrids of okra (Abelmoschus esculentus (L.) Moench). Madras Agric. J. 90 (4 - 6) : $322-325$.

Kumbhani R. P., P. R. Godhani and R. S. Fougat. 1993. Hybrid vigour in eight parent diallel cross in okra. Gujrat Agricultural Uni. Res. J. $18(2): 13$ - 18.

Machado C. F., J. B. Santos, G. H. Nunes and M. A. P. Ármalo. 2002. Choice of common bean parents based on combining ability estimates. Genetics and Molecular Biology $25: 179-183$.

Metha N., B. S. Asati and S. R. Mamidwar. 2007. Heterosis and gene action in okra. Bangladesh J. Agril. Res. 32 (3) : 421 - 432.

Murtaza N. 2005. Study of gene effects for boll number, boll weight and seed index in cotton. Journal of Central European Agriculture 6 (3) : 255 - 262.

Nimbalkar C. A., U. T. Desai and S. G. Bhalekar. 2004. Heterosis studies in okra. Journal of
Maharashtra Agricultural Universities 29 (3) : 360 - 362.

Poshiva V. K. and P. T. Shukla. 1985. Correlation coefficient between per se performance, array mean and general combining ability effects in okra (Abelmoschus esculentus L. Moench). Gujt. Agril. Univ. Res. J., 11 (1) : $53-54$.

Rajani B., P. Manju, P. N. Manikantan and P. Saraswathy. 2001. Combining ability in okra (Abelmoschus esculentus (L.) Moench). Journal of Tropical Agriculture 39 : 98 - 101.

Schnell F. W. and H. C. Becker. 1986. Yield and yield stability in a balanced system of widely differing population structures in Zea mays L. Plant Breeding 97 : 30 - 38.

Shivagamasundari S., I. Irulappan, R. Arumugam and S. Jayasankar. 1992. Combining ability in okra (Abelmoschus esculentus (L.) Moench). South Indian Hort. $40: 21$ - 27.

Shukla A. K., N. C. Gautham, A. K. Tiwari and A. K. Chaturvedi. 1989. Heterosis and combining ability in okra (Abelmoschus esculentus (L.) Moench). Vegetable Sci. 16 (2) : 191 - 192.

Viana J. M. S. 2000. The parametric restrictions of the Griffing diallel analysis model : combining ability analysis. Genetics and Molecular Biology 23 (4) : 877 - 881.

Viana J. M. S. and F. D. P. Matta. 2003. Analysis of general and specific combining abilities of popcorn populations, including selfed parents. Genetics and Molecular Biology 26 (4) : 465 - 471.

Wright J. W. 1964. L'hybridation entre espèces et races. Unasylva 73 - 74 (18) : 49 - 62. 